\title{
Implementing a Business Intelligence Environment in Local Market of Pakistan
}

\author{
FARIA JAMEEL*, FARIDA MEMON**, PERVEZ SHAIKH***, AND NAFEES AHMED MEMON*** \\ RECEIVED ON 17.03.2016 ACCEPTED ON 16.08.206 \\ ABSTRACT
}

\begin{abstract}
The BI (Business Intelligence) has gained great success during the last decade throughout the world to aid in decision support with the availability of necessary knowledge to reduce costs, increase revenues and minimize risks. The local market of Pakistan is still not very much aware of its benefits, except some multinationals which are using these tools since almost 7-8 years and earning more revenues and improved performances and a few others are under the process of implementation. The small and medium sized businesses of our local market are focused for the implementation of BI. The pros and cons are identified by analysing the BI tools being used by other large companies here in Pakistan and feasibility of these tools at small and medium enterprises is discussed so that they too may focus on their KPIs (Key Performance Indicator) to increase their performance level.
\end{abstract}

Key Words: Business Intelligence, Online Analytical Processing, Data Warehouse, Data Mart, Extract, Transform and Load.

\section{INTRODUCTION}

$\mathrm{B}$ usinesses have always been in a need of organizing their information/data to get the best benefit out of it [1]. The idea of BI came up as a solution to the businessmen. Especially with the fast growing world of business in the 21st century made the BI an unavoidable move. But the use of the BI tools gradually came up with a few areas to be explored more and to be worked upon in more detail [2]. The earlier decision support system had to be modified according to the modern needs. BI took many turns and it also incorporated the techniques of data warehousing, data mining and the latest issue identified has now moved on to the operational data sores and knowledge management/content management in order to make better strategic decisions [2].

Sufficient amount of information has become a requirement and need of a human being in order to succeed in today's environment. The information needs to be integrated from multiple sources and adjusted to a certain level. The structure of sources and its amount may change with time and everything is needed to be available for decision making at each individual's dashboard in the up-to-date form.

\footnotetext{
* Department of Computer Science, Zulfiqar Ali Bhutto Institute of Science \& Technology, Karachi.

** Department of Electronic Engineering, Mehran University of Engineering \& Technology, Jamshoro.

*** Department of Civil Engineering, Mehran University of Engineering \& Technology, Jamshoro.
}

Mehran University Research Journal of Engineering \& Technology, Volume 36, No. 2, April, 2017 [p-ISSN: 0254-7821, e-ISSN: 2413-7219] 
BI can be defined as the process of turning data into information and then into knowledge [3]. It was born in 1990's to support decision making and to better understand the key factors involved in the decision making.

During the last decade, the trends in BI have changed dramatically and companies started using BI to achieve their goals focusing on the strategies based on metricsdriven data warehousing [3]. Unfortunately, Data warehousing failed in top-down enforcement of company's strategies though supporting the bottom-up extraction of information from data. Hence, a new approach emerged which is called Business Performance Management. With the assistance of knowledge implementation and business process reuse, decision making efficiency of BI system can be improved when dealing with new tasks [4]. BI became a popular term in the business and IT (Information Technology) communities only in the 1990s [5]. In the late 2000s, business analytics was introduced to represent the key analytical component in BI according to Davenport in 2006.

In order to improve the efficiency of a business using a BI system, data is transformed into useful information and knowledge management techniques are applied to manage information and support decision making. An 'intelligent enterprise' is the one that uses BI to advance its business by predicting how future economic and market changes will affect its business [4]. The information collected and integrated from multiple sources is required to support the process of decision making. Amount and structure of sources can change with time and the level of detail can also be varied as well [6]. Application of BI tools was connected with data warehouse as a necessary prerequisite for the data mining as a further extension.
In this study, our local market has been focussed for BI implementation and the factors involved in the selection of tools have been analysed. The implementation strategies are then mentioned at general and specific levels and need of local market is identified for implementing a BI environment.

\section{BACKGROUND/OVERVIEW}

The quality of a company's information system has become recognized as a strategic corporate advantage. Information systems have become the backbone of the modern businesses and as such are crucial to its functioning. An organization with the appropriate information tools can take advantage of business opportunities quickly and can adapt itself to changing business requirements and needs. Many solutions have come to help with this problem. Products, techniques and services that help overcome these problems can be critical to the success of the business itself. Business environment have been changed dramatically during the last decade or so and it has also changed the needs of the workforce as well. They need more informed and organized knowledge to make better decisions. The availability of a wide variety of BI solutions has also made the businesses become more successful by increasing their revenues and ROI (Return on Investment).

\subsection{Informed Decisions}

As the companies became more dependent on the information-processing capability, the same growth of dependence put stress on that capability itself. With the expansion in business processes and the ultimate junk of information, now the companies need to filter out the information for making better decisions [4]. Today, the companies and executives are asking IT vendors for new tools capable of handling new business scenario [3]. 
With the change in time and the change in business scenario, the managers need to take more risky and informed decisions in order to stay ahead in the competitive market. Many solutions have come up to solve this problem and some have even gone. Some of them are difficult to integrate with the existing technologies e.g. Client/Server technologies and distributed computing are hopeful solutions but are hard to integrate. BI tries to cover all the deficiencies of the tools and information systems developed earlier and to meet the demands of the current business scenario which needs to have information available in the form of knowledge in order to make informed decisions.

\section{BUSINESS INTELLIGENCE IN LOCAL MARKET}

BI is a phrase coined by Howard Dresner of Gartner Group in 1989. It encompasses all the capabilities required to turn data into intelligence that everyone in the organization can trust and use for more effective decision making [4]. BI came into existence in the early 90 's to satisfy the manager's needs for efficiently analysing the business data to understand their business in a better way and to make better decisions. Nowadays, most of the BI tools are based on DW (Data Warehouse) and data analysing software, usually OLAP (Online Analytical Processing). It makes decisions by relying on the data analysis reports.

An 'intelligent' business is the one who can use BI tools to better predict how future economic and market changes will affect its business [4]. In BI, data is organized in such a way that report formation becomes very simple.

\subsection{Issues with BI Implementation}

$\mathrm{BI}$ works in conjunction with a proper OLAP engine. The OLAP engine must be supported by a DW which is a restructured way of data. In order to have an overview of basic information related to customer, product and performance, the simplest solution is to place information in one location and use it from this location for further analysis and reporting. Such solutions are known as DW [6]. If DW is not required in the business, at least a data mart must be in place.

Data storage is another issue. Data are stored in different formats at different places and in different type of databases. All the data must be consolidated to form a consistent business views that support the approach used for delivery [4].

\subsection{Uses of BI in Local Market}

The decade of the 2010s promises to be an exciting one for high-impact BI\&A (Business Intelligence \& Analytics) research and development for both industry and academia throughout the world [5]. BI may be useful in large or medium sized businesses where there is a huge amount of data to be handled at the backend. It helps in decision making by combining itself to the DW and providing every kind of report at just a click of a mouse [7].

It may be used in Trend analysis and for cross-selling of any two products, for making comparisons, e.g. Suppose Lever Brothers want to compare the trend of Lipton and Supreme to make future promotion policy. BI is the best tool for such a situation [8]. Previously businesses used ERPs (Enterprise Resource Planning) and have moved on to SAP now [9]. The BI may be helpful in fetching any of the two parts of a DW, i.e. transaction data which is collected on daily, fortnightly or monthly basis, and the reference data for linking customers, suppliers, vendors or even products [10].

It also tells the users whether a particular promotion campaign was useful or not and the business can focus on any particular area or region for future. Suppose a mobile phone company wants to analyse if SIMs sent to

Mehran University Research Journal of Engineering \& Technology, Volume 36, No. 2, April, 2017 [p-ISSN: 0254-7821, e-ISSN: 2413-7219] 
Balakot benefits their business or not. Pharmaceutical companies may get the data from government authorities to analyse the trend of any particular drug.

It reduces time consumed in decision making by providing up-to-date information according to the specific needs of individual users. It also improves the quality of decisions by making all the updated information available on the dashboard of individual users [8]. The users get aware of each and every detail of happenings in the organization. It is defined as getting the right information to the right people at the right time.

Presentation is significant in BI [8]. Dashboards are created, relevant panels are created. Different users may want different combination of panels and BI provides easy to make creation of their own dashboards. Pivot reports and Score boards can be created within no time. The only thing required is the right combination of OLAP tool and a BI tool. Businesses can pick any customer and see if he or she is making payments or not and in this way, you may know your customer better [10]. Sales people can analyse why there is an increase or decrease in Sales.

Shell Oils also makes comparative analysis using BI tool to see if any particular marketing campaign was successful or not. They also compare their performances before and after any particular strategy to focus if that particular strategy was successful or not [10].

\subsection{Comparison between BI and MIS- for Local Market Users}

Business have to bear upfront cost to implement $\mathrm{BI}$ in order to architect DW or Data Mart, create ETL (Extract, Transform and Load) process, transfer data from Data Mart and create cycle/batch for smooth data processing. Once it is implemented, it delivers every report very quickly making decision making process efficient and reliable [8].
MIS may be cost effective but it works in slow fashion. Suppose the time limit to finish a report is 2 months, then by the end of two-month time, some reports may become obsolete or outdated as per the requirement of the report. This is called data latency, which is the interval or gap between the time an event occurs and the time it is perceived by the user [3].

\subsection{Why Need BI in Pakistan}

$\mathrm{BI}$ is needed to make the decision making process more effective. As the businesses have expanded so much during the recent times, the need of having all the relevant information available on desk to make in-time decisions has increased. Different companies across the world are working to solve this issue and many different types of tools and softwares have also been developed and the process is still going on in order to make it more easy to use and convenient.

BI concept actually came into the market in 1990's and businesses have been taking advantage of the BI tools and vendors have been taking the feedback to make more improvements. The trends moved from Customer Relationship Management to Business Process Management and Knowledge Management. But still BI tools have been widely used in enterprises throughout the world to improve ROI and profitability.

Market in Pakistan in general is still not very much aware of the benefits of BI. They don't know what benefits they can get by using $\mathrm{BI}$ and how they can raise their revenues by using the IT properly. In most of the local organizations, data is not organized well and the organizations are not able to make the best decisions out of the information they have got.

There are very limited organizations locally who are aware of BI and are taking advantage of it e.g. State Bank of

Mehran University Research Journal of Engineering \& Technology, Volume 36, No. 2, April, 2017 [p-ISSN: 0254-7821, e-ISSN: 2413-7219] 
Pakistan, Unilever, Mobilink, Pakistan Petroleum and there are a few who are under process of implementation e.g. KESC (Karachi Electric Supply Corporation), SBL (Soneri Bank Limited) and HBL (Habib Bank Limited). If BI is implemented in our local businesses, then it may improve their returns and in turn improve the economy of the country overall.

\section{BUSINESS INTELLIGENCE TOOLS IMPLEMENTED IN LOCAL MARKET}

After conducting interviews with the BI experts and the users in their mentioned organizations, the list of BI tools was organised which are being used at particular organizations in Pakistan.

\subsection{Cognos}

It is a BI solution by IBM (Institute of Business Management) and is implemented at Shell Oil (Pvt) Limited, Pakistan. Cognos is implemented there since more than nine years and they are running it successfully at their client side [9]. Its analytical capabilities have improved their performance and sales. The main use of Cognos as a BI tool at Shell Oils is at Sales side where they have million transactions to handle monthly. They can very easily analyse the trends, make comparisons and make better decisions [8].

It is a reporting tool and it contains OLAP engine as well. It offers Performance Management, Scorecarding, Budgeting, forecasting and consolidation. It helps in aligning tactics with strategy. It is a very successful BI tool throughout the world and many success stories are also available in the different industries of international market. IBM Cognos software is used in commercial banks to increase profits, improve accuracy, reduce credit risk, segment customers more effectively, and cut reporting time from three weeks to two hours [11].

\subsection{Business Objects}

It is implemented at Unilever and they are taking full advantage of it by getting analytical reports in any required format and in a much reduced time as compared to the previous system according to a worker at Unilever. Different departments get the customized benefit out of Business Objects, e.g. the sales people use it in their own way, the finance department has its own requirements of data analysis.

Using Business Objects, decision-makers are empowered with flexible ad hoc reporting, query, analysis, and dashboards via an intuitive web interface that enables fast, flexible decision-making — all without relying on IT [12]. It is an ideal choice for those organizations which need to deliver query and analysis and enterprise operational reports documents over the web to smaller audiences. Users don't have to deal with multiple servers, and complex configurations.

\subsection{Mondrian}

Mondrian is an open source OLAP server written in Java. Mondrian provides the interactive ability to analyze very large datasets stored in SQL databases without having to write SQL and is used for multi-dimensional exploration of data. It also supports the translation of MDX (Multi Dimensional Expression) language into SQL which is needed for proper BI implementation. It is not very featured rich and not very advisable.

Any application of Mondrian in the local market could not be found but it may be considered as a cost effective option by the local customers because of it being an open source tool [13].

\subsection{QlikView}

A local vendor AbbuSys provides this BI solution with the collaboration with QlikTech International. Recently

Mehran University Research Journal of Engineering \& Technology, Volume 36, No. 2, April, 2017 [p-ISSN: 0254-7821, e-ISSN: 2413-7219] 
they have implemented it at SBL and their deal with Habib

Bank Limited was under process till the writing of this document [1].

QlikView brings fast results and better decision making to a wide range of customers across the world and many of the success stories of their clients have been published on their website as well like others. They claim that the revenue goes up to $>\$ 1 \mathrm{~B}$ for large businesses and up to $\$ 100 \mathrm{M}-\$ 1 \mathrm{~B}$ in medium businesses after using their BI solution QlikView [8].

It connects data coming from any of the multiple sources to the SQL (Structured Query Language) server to serve as the backend tool. Then OLAP connects the backend tool with their frontend BI solution [1].

\subsection{Server Analysis Services}

SQL SSAS (Server Analysis Services) is implemented at UBL. It provides a unified and integrated view of all your business data as the foundation for all of your traditional reporting, OLAP analysis, KPI scorecards, and data mining.

Analysis Services provides a UDM (Unified Dimensional Model) which defines business entities, logic, calculations, and metrics. It is a central place that serves as the single version for all reports, spreadsheets, OLAP browsers, KPIs, and analytical applications. Proactive caching of SSAS enables the combination of real-time updates with MOLAP (Multidimensional Online Analytical Processing) perform as per the business requirements. Its KPI framework provides a rich centralized repository defining key metrics and scorecards.

Organizations can easily build applications like multilingual translations, MDX scripts, BI wizards and Data Mining etc.

\subsection{Oracle BI Suite}

It is the top ranked BI tool and is planned to be implemented at the State Bank of Pakistan according to the ISTD at SBP (State Bank of Pakistan). They are currently using Oracle Discover and they are strongly in favor of Oracle BI suite because of its strong database support.

The OBIEE Plus (Oracle Business Intelligence Suite Enterprise Edition Plus) is designed to meet this evolving business need. It is a comprehensive suite of BI tools. OBIEE Plus comprises powerful BI server technology and BI presentation tools - referred to as Oracle BI Enterprise Edition [14].

It also offers excellent financial reporting, web analysis, and interactive reporting tools embedded into it.

\subsection{MS Share Point}

SharePoint is implemented at Mobilink and Pakistan Petroleum and Microsoft itself. KESC is also planning to implement it as a BI tool in their organization [12].

MS Share Point is also considered to be a BI tool though it has limited capabilities as compared to other BI tools. It is more like a Content Manager which manages different types of content coming from multiple sources from within and outside the organization [8]. It organizes the data very well and presents it in a way required by individual users. The feature it lacks as a BI tool is the reporting side which is the main feature of BI. State Bank of Pakistan is under the process of implementing a document management system and they are considering MS SharePoint as an option.

Share Point may serve as a delivery platform for BI. It gathers information from multiple sources and provides information at one place. It also creates dashboards according to the particular needs of the users providing

Mehran University Research Journal of Engineering \& Technology, Volume 36, No. 2, April, 2017 [p-ISSN: 0254-7821, e-ISSN: 2413-7219] 
the whole information regarding their business using its end-user delivery mechanism [13]. It even has KPI dashboards which may be useful for BI implementation.

The BI strategy of Microsoft comprises a suite of server and client-side data integration tools. As shown in Fig. 1, the analytical and reporting tools in SQL Server 2005 provide the backbone data management infrastructure, while Microsoft Office applications, specifically Microsoft Office Excel, provide the flexibility for information workers to remotely interact with centralized and secure data sources [15].

Excel, Excel Services, SharePoint Report Center, and the BDC (Business Data Catalog) are the core, BI features within the Office suite of products. SQL Server 2005 Reporting Services, Analysis Services, and PerformancePoint Server 2007 extend the core BI functionality by offering enhanced reporting capabilities, data warehousing, in-depth data analysis, and real-time monitoring [16].

\section{IMPLEMENTATION STRATEGIES}

\subsection{General Strategies}

For implementation, data mart must be in place, OLAP must be in place and proper integration of both is utmost important [8].

Reporting front-end must be at its place for all executive users. The roles of all the users must be defined so that proper users may see proper reports needed for their managerial level. BI technicians are needed who can create $\mathrm{BI}$ reports on the fly and a Business analyst is also needed for implementing BI so as to identify what sort of reports are to be generated [8].

Further extension of BI goes to data mining. It gives the data density. Suppose sales are increased but the user doesn't know the reason why. Different factors are introduced and data mining helps to identify the data density in that particular business using all the factors using visualization.

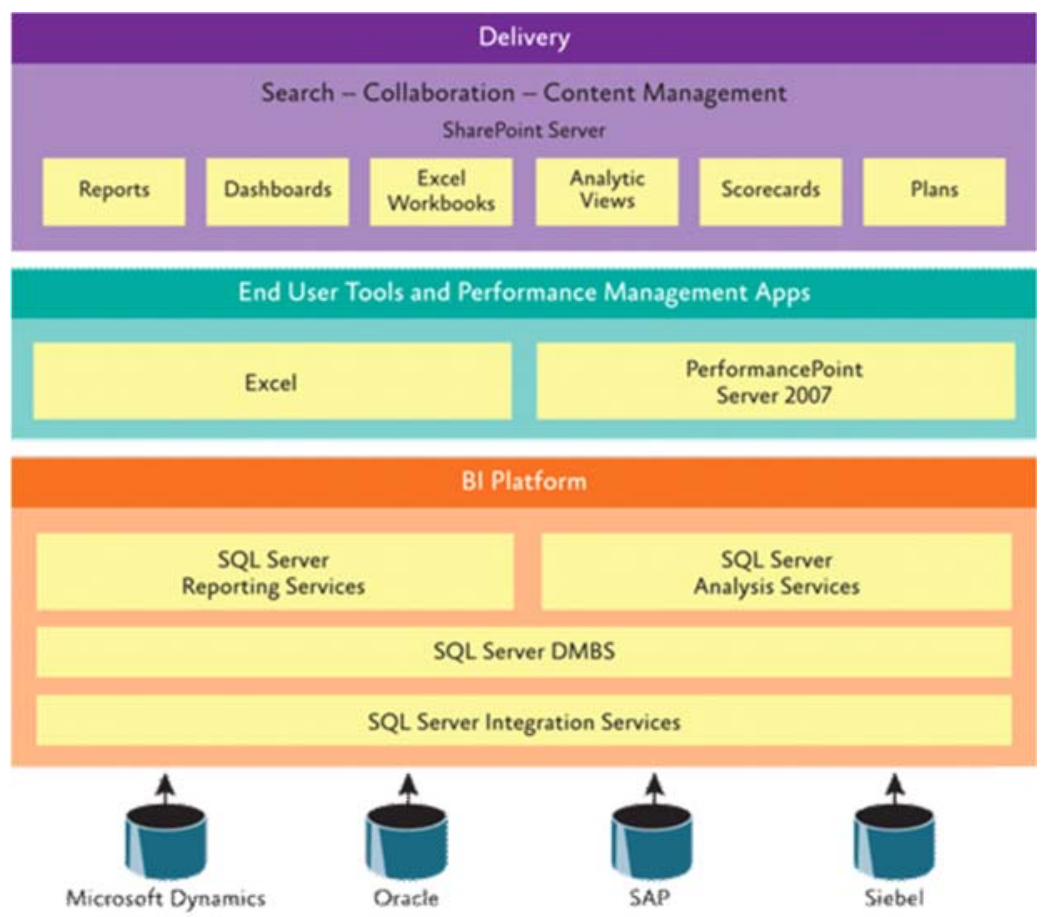

FIG. 1. ARCHITECTURE OF THE INTEGRATED MICROSOFT BI SOLUTION [8] 
Usually, the disparate data don't speak to each other, and each might be managed by different groups that report to different organizational entities [4]. Data quality has to be considered after the data has been identified and collected correctly. It would be a big mistake if this point is ignored.

\subsection{Specific Strategies for Local Markets}

Focusing on our local markets, the data must be cleansed and organized into a data warehouse or at least a data mart. Then it must be linked to a query language such as SQL since it is used as the backend tool in most of the different BI tools. All the steps discussed above are very essential for them to take the benefits of using BI and improve their analytical skills, planning and decision making.

Local vendors like AbbuSys may be consulted for BI support but they too claim that $\mathrm{BI}$ is not suitable for small businesses because of its complex backend requirements which may increase the overall cost to take the best advantage out of it.

Open source BI tools like Mondrian are also available to be considered as a cost effective solution but they have not been proved to be very successful BI tools through any source [8].

\section{NEED OF LOCAL MARKET (RESEARCH FINDINGS)}

The local market of Pakistan majorly comprises of medium and small businesses. These businesses have limited budget and cannot afford to go for most of the BI tools available in the market. The BI tools are very much efficient and may help in uplifting the businesses if the proper implementation strategy is followed. It is comparatively a complex and lengthy process to fully implement the BI tool in an organization. It is useful for those companies who can afford and are running large businesses, having a huge database at the backend. These companies have varied preferences regarding BI tools according to their own requirements. But unfortunately, the success stories available in our local market are only limited to the multinationals which could not attract more companies opt for it.

Some BI tools have scalability and manageability limitations that make them expensive to maintain as the amount of data, the number of data sources, the number of active users, and the complexity of the analysis being performed grows. As a result, executives find that despite their continued investment in BI technology, they still do not have the relevant information they need to make decisions quickly and effectively.

Many local organizations do not even have their data warehouse set up and even if they want to go for Business Intelligence, they need to incur cost for data warehouse first and for that, they need to do the data cleansing as well because if the data warehouse is not in place then the data must not be organized and may even have redundancy in it. After the implementation, they would also have to bear additional training costs as well. Therefore it becomes a long and complicated process and is not at all cost effective for the small and medium sized businesses of our country.

The survey of the local market tells us that many of these businesses are using MS Excel to analyze past and future trends using 'what-if analysis' and 'pivot table' features and MS Office overall is very commonly used at almost every level. Therefore if we combine the features of MS Share Point and MS Excel, the medium and small sized businesses may take advantage of it and now with the merger of MS Share Point and MS Performance Point, they can get the full advantage of BI environment. It will be a cost effective solution for them since they are already

Mehran University Research Journal of Engineering \& Technology, Volume 36, No. 2, April, 2017 [p-ISSN: 0254-7821, e-ISSN: 2413-7219] 
using MS excel and are well familiar with the Microsoft environment, so they will not need any specialized training.

If Excel sheets may be refreshed dynamically at the backend, they may be reflected in Share Point portals and businesses may get up-to-date information available on the dashboards of Share Point as shown in Fig. 2. If a business has a DW or a data mart at the backend, it may be linked through SQL and Excel may serve as the reporting tool at the front end [13].

Microsoft has its offices and has many certified business partners in the main cities of Pakistan and they provide full-time support for their product implementation. Whereas other companies like Oracle, Siebel and IBM does not have so much customer support in our local market except some software houses providing services on BI to name a few e.g. Abusyss. Mobilink has made its HR portal using Share Point and so has the Pakistan Petroleum Services.
Even Microsoft itself does its BI management worth $\$ 60$ billion using Excel and is successfully increasing its revenues and ROIs [13].

In future, they plan to develop BI solutions that are affordable and easily accessible with a view to furthering the vision of $\mathrm{BI}$ for the masses.

\section{CONCLUSIONS}

Different BI tools were discussed which are popular and being used in the local industry as well. Unfortunately, no big success stories are available in terms of performance or ROI in business organizations related to actual BI tools implementation and around 70-80 percent of businesses are still using Excel for their analysis and forecasting purpose. Though the big companies are running BI successfully but the reason maybe perhaps in our local markets usually businesses do not keep track of the statistics showing the comparison between the pre and post implementation performance. Most of the

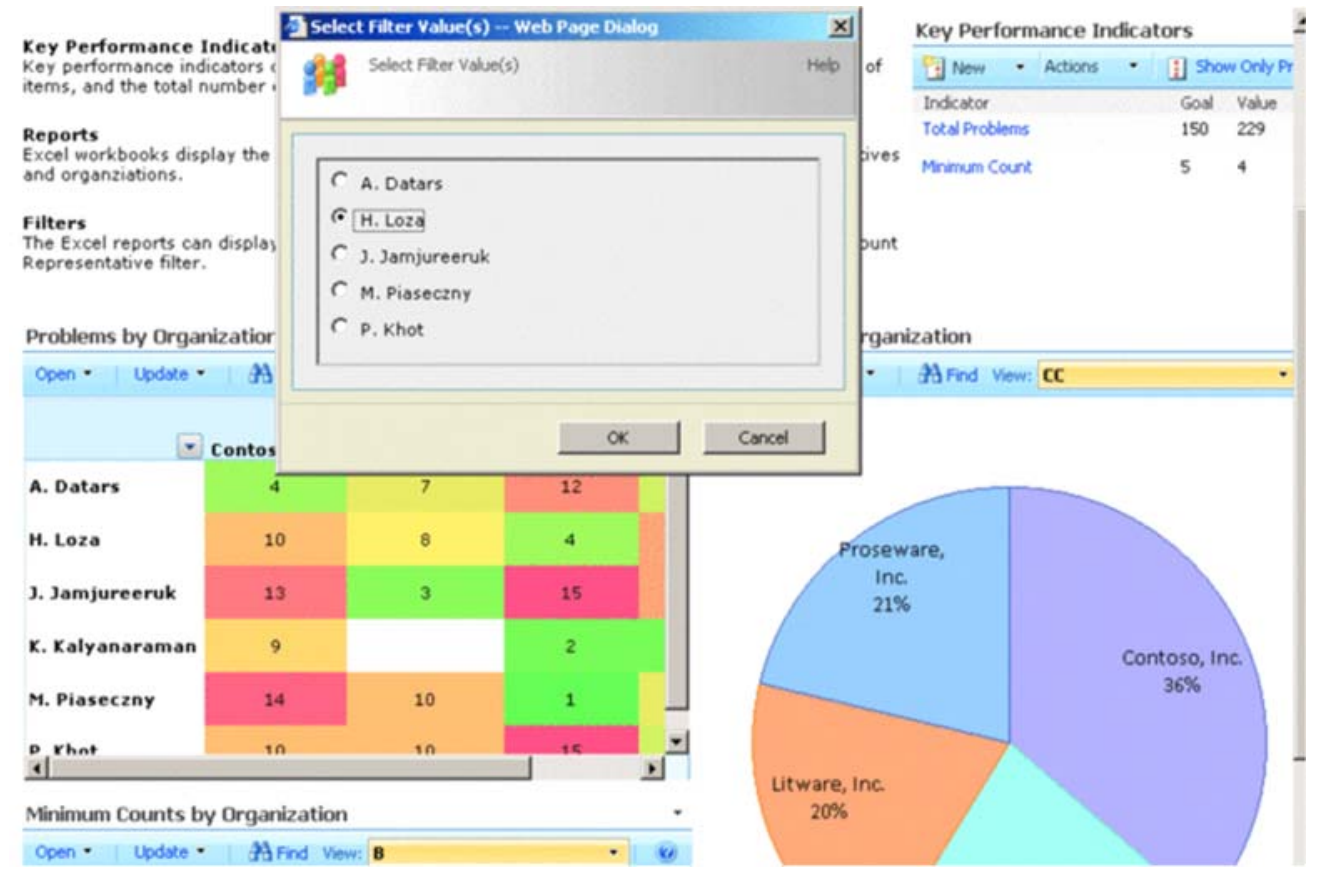

FIG. 2. DASHBOARD OF NEW SHAREPOINT

Mehran University Research Journal of Engineering \& Technology, Volume 36, No. 2, April, 2017 [p-ISSN: 0254-7821, e-ISSN: 2413-7219] 
businesses are not willing to put so much money into BI implementation and many of the small and medium sized businesses even cannot afford to do so. Therefore, SharePoint Server is considered as a solution for the small and medium sized businesses of our local market.

SharePoint Server is based on the common MS Office environment which most of the businessmen are familiar with. They can continue working on Excel and integrate it with SharePoint. Microsoft has now integrated SharePoint Server with PerformancePoint Server to make it a more powerful BI tool containing all the basic features of BI and it is expected to capture the masses since it does not have to depend on any other environment and therefore will be a cost effective solution.

SharePoint Server is based on the common MS Office environment which most of the businessmen are familiar with. They can continue working on Excel and integrate it with SharePoint. Microsoft has now integrated SharePoint Server with PerformancePoint Server to make it a more powerful BI tool containing all the basic features of BI and it is expected to capture the masses since it does not have to depend on any other environment and therefore will be a cost effective solution.

\section{ACKNOWLEDGEMENTS}

Authors would like to acknowledge Sayed Yousuf, CTO, Progressive Systems, Pakistan, Mr. Zafarul Islam, Technology Specialist, Infrastructure \& Security Microsoft Corporation, Pakistan, Mr. Sadiqain Khoja, Shell Oil, Pakistan, Mr. Zainul Abedeen, Technical Solution Manager, AbbuSys (Pvt.) Limited, for their insightful interviews.

\section{REFERENCES}

[1] Abedeen, Z., "Interviewee, Technical Solutions Manager", AbbuSys (Pvt) Limited, Pakistan [Interview] $15^{\text {th }}$ March, 2014. Support in Business Intelligence System", Proceedings of $1^{\text {st }}$ International Multi-Symposiums on Computer and Computational Sciences, 2006.

[3] Golfarelli, M., Rizzi, S., and Cella, I., "Beyond Data Warehousing: What's Next in Business Intelligence?", DOLAP, Washington DC, USA, 2004.

[4] Bogza, R.M., and Zaharie, D., "Business Intelligence as a Competitive Differentiator", IEEE International Conference on Automation, Quality and Testing, Robotics, Volume 1, pp. 146 - 151, 2008.

[5] Hsinchun, R.H.L.C.V.C.S., "Business Intelligence and Analytics: From Big Data to Big Impact", MIS Quarterly, Volume 36, No. 4, pp. 1165-1188, December, 2012.

[6] Tvrdíková, M., "Support of Decision Making by Business Intelligence Tools", 6th International Conference on Computer Information Systems and Industrial Management Applications, 2007.

[7] "www.businessintelligence.com" 9 $9^{\text {th }}$ April 2015 [Online] Available: www.businessintelligence.com [Accessed $9^{\text {th }}$ April, 2015].

[8] Yousuf, S., "Interviewee", CTO, Progressive Systems, Pakistan [Interview] 16 $6^{\text {th }}$ March, 2014.

[9] "Wikepedia", 12 $2^{\text {th }}$ May 2014. [Online] Available: www.wikepedia.com,en.wikipedia.org/wiki/ Business_intelligence, en.wikipedia.org/wiki/ Online_analytical_processing, en.wikipedia.org/wiki/ Data_warehouse [Accessed $12^{\text {th }}$ May, 2014].

[10] Sadiqain Khoja, Interviewee, Shell Oil, Pakistan [Interview] 19 $9^{\text {th }}$ August, 2012.

[11] www.cognos.com, 2015 [Online] Available: http:// www.cognos.com/solutions/industry/banking-financialservices/customers.html [Accessed 25th August, 2015].

[12] "Business Objects Crystal Decisions QuickStart Guide", [Online] Available:www.calpolycorporation.org/docs/ finance/BOEInfoViewQuickStart.pdf [Accessed $16^{\text {th }}$ November, 2015].

[13] Islam, Z., "Interviewee", Technology Specialist, Infrastructure \& Security Microsoft Corporation, Pakistan. [Interview] 2 $2^{\text {nd }}$ September, 2014.

[14] Oracle, "Oracle Business Intelligence Suite Enterprise Edition Plus -Technical Overview", An Oracle White Paper, November, 2007.

[15] B.C.A.B.E., Microsoft SharePoint Teams, "Business Intelligence with SharePoint and Excel", TechNet Magazine, February, 2009.

[16] www.microsoft.com, Microsoft, $15^{\text {th }}$ December, 2015 [Online] Available: www.microsoft.com/BI/ [Accessed $15^{\text {th }}$ December, 2015]. 